\title{
Effect of Valve Height on the Opening and Closing Performance of the Aortic Valve Under Aortic Root Dilatation
}

\author{
Qianwen Hou 1,2, Guimei Liu 1,2, Ning Liu 1,2, Honghui Zhang 1,2, Zhuoran Qu ${ }^{1,2}$, \\ Hanbing Zhang ${ }^{1,2}$, Hui $\mathrm{Li}^{1,2}$, Youlian Pan ${ }^{1,2}$ and Aike Qiao ${ }^{1,2 *}$
}

${ }^{1}$ Faculty of Environment and Life, Beijing University of Technology, Beijing, China, ${ }^{2}$ Intelligent Physiological Measurement and Clinical Translation, Beijing International Base for Scientific and Technological Cooperation, Beijing, China

OPEN ACCESS

Edited by:

Zhiyong $L i$

Queensland University of

Technology, Australia

Reviewed by:

Shengzhang Wang,

Fudan University, China

Dominik Obrist

University of Bern, Switzerland

Changyan Lin,

Capital Medical University, China

*Correspondence:

Aike Qiao

qak@bjut.edu.cn

Specialty section:

This article was submitted to

Computational Physiology and

Medicine,

a section of the journal

Frontiers in Physiology

Received: 19 April 2021

Accepted: 04 August 2021

Published: 30 August 2021

Citation:

Hou Q, Liu G, Liu N, Zhang H, Qu Z, Zhang H, Li H, Pan Y and Qiao A (2021) Effect of Valve Height on the Opening and Closing Performance of the Aortic Valve Under Aortic Root Dilatation. Front. Physiol. 12:697502. doi: 10.3389/fphys.2021.697502
Patients with aortic valve disease can suffer from valve insufficiency after valve repair surgery due to aortic root dilatation. The paper investigates the effect of valve height (Hv) on the aortic valve opening and closing in order to select the appropriate range of $\mathrm{Hv}$ for smoother blood flow through the aortic valve and valve closure completely in the case of continuous aortic root dilatation. A total of 20 parameterized three-dimensional models of the aortic root were constructed following clinical surgical guidance. Aortic annulus diameter (DAA) was separately set to $26,27,28,29$, and $30 \mathrm{~mm}$ to simulate aortic root dilatation. $H_{v}$ value was separately set to $13.5,14,14.5$, and $15 \mathrm{~mm}$ to simulate aortic valve alterations in surgery. Time-varying pressure loads were applied to the valve, vessel wall of the ascending aorta, and left ventricle. Then, finite element analysis software was employed to simulate the movement and mechanics of the aortic root. The feasible design range of the valve size was evaluated using maximum stress, geometric orifice area (GOA), and leaflet contact force. The results show that the valve was incompletely closed when $H_{V}$ was $13.5 \mathrm{~mm}$ and $D_{A A}$ was 29 or $30 \mathrm{~mm}$. The GOA of the valve was small when $H_{V}$ was $15 \mathrm{~mm}$ and $D_{A A}$ was 26 or $27 \mathrm{~mm}$. The corresponding values of the other models were within the normal range. Compared with the model with an $\mathrm{H}_{V}$ of $14 \mathrm{~mm}$, the model with an $\mathrm{H}_{\mathrm{V}}$ of $14.5 \mathrm{~mm}$ could effectively reduce maximum stress and had relatively larger GOA and less change in contact force. As a result, valve height affects the performance of aortic valve opening and closing. Smaller $\mathrm{H}_{V}$ is adapted to smaller $D_{A A}$ and vice versa. When $H_{V}$ is $14.5 \mathrm{~mm}$, the valve is well adapted to the dilatation of the aortic root to enhance repair durability. Therefore, more attention should be paid to $H_{V}$ in surgical planning.

Keywords: aortic valve, aortic root dilatation, aortic valve repair, biomechanics, numerical simulation

\section{INTRODUCTION}

The aortic root consists of the sinuses of Valsalva, aortic valve, aortic annulus (AA), aorto-ventricular junction, and sinotubular junction (David, 2013). The aortic valve controls unidirectional blood flow from the left ventricle to the aorta by performing a regular opening and closing movement with contraction and relaxation of the heart. Aortic valve insufficiency (AI) and aortic stenosis (AS) represent the most common aortic valve diseases (Alkhodari and Fraiwan, 2021; Wazaren et al., 2021; Zhang et al., 2021). AI causes the blood to flow back into the left 
ventricle, leading to left ventricular dysfunction and even diastolic heart failure. As for AS, patients with moderate to severe AS can develop obstruction of the left ventricular output and reduction in cardiac output, which may cause myocardial insufficiency, angina pectoris, and even sudden death. Therefore, it is essential to guarantee a normal function of the aortic valve.

For young patients who suffer from aortic valve dysfunction, it is challenging to decide on the choice of surgical procedure. For example, mechanical valve replacement typically necessitates lifelong anticoagulant therapy, which increases the risk of hemorrhage and thromboembolism and decreases the quality of life of patients. On the other hand, biologic prosthetic valves have a higher risk of reoperation compared with mechanical valves due to lack of structural durability, which leads to a significant increase in mortality (Natalie et al., 2016; Goldstone et al., 2017). In the past two decades, aortic valve preservation or repair operations have become increasingly popular alternatives to aortic valve replacement for tricuspid and bicuspid valves. In young patients, valve repair is more likely to provide better quality of life and fewer valve-related complications compared with prosthetic valve; however, this has to be balanced against the risk of reoperation (Schäfers et al., 2013; Arabkhani et al., 2015; Lansac et al., 2015; Emmanuel et al., 2016; Ravil et al., 2019).

In clinical practice, gradual expansion and deformation of the aorto-ventricular junction have been observed as the age of patients increase, resulting in an increase in aortic annulus diameter $\left(D_{A A}\right)$. An untreated dilated aortic annulus of more than $25-28 \mathrm{~mm}$ can result in aortic regurgitation (AR) or AS, representing a major risk factor for the failure of aortic valve repair operations (Aicher et al., 2012; Navarra et al., 2013; Laurent et al., 2014). In such cases, reoperation is required to restore the normal occlusion of the aortic valve. Therefore, before the initial repair operation in young patients, physicians should consider that the dilatation of the aortic root can cause reappearance of the valve dysfunction and limit the durability of the repair to a few years after the operation. Hence, determining the geometric size design of the aortic valve to adapt to aortic root dilatation and enhance repair durability is a key challenge.

As a functional unit, the geometric interrelation between the aortic valve and root has led to realizing that the reconstruction of near-normal valve and root geometry is essential to obtain a good functional repairing result. For surgeons, it is necessary to design a specific valve according to requirements when functional dimensions are restored in the leaky valve of a patient (Pan et al., 2014). Previous studies have been done on the influence of aortic root geometry on valve closure performance to evaluatethe aortic valve sparing surgery before the operation, since the numerical models feature numerous advantages over the attempts of surgeons. Further studies (Gnyaneshwar et al., 2002; Howard et al., 2003; Weltert et al., 2013) constructed finite element models to perform simulation studies, and their results revealed that the closing performance of the aortic valve could be affected by increase in the size of the aortic root. Besides, they reported that the expansion of the aortic root was the main factor leading to increased pressure of the leaflets and then to AI. By numerical simulation, Marom and colleagues formulated six idealized models of the aortic root separately with a $\mathrm{D}_{\mathrm{AA}}$ of 20 and $30 \mathrm{~mm}$. The results proved that the changes in $\mathrm{D}_{\text {AA }}$ produced a significant inhibitory effect on aortic valve performance (Marom et al., 2013). Additional simulation results indicated that increasing leaflet graft height leads to an increase in the amount of growth that the reconstructed valve can accommodate. Furthermore, for a given vessel size, an increased valve height $\left(\mathrm{H}_{\mathrm{V}}\right)$ leads to better coaptation metrics (Hammer et al., 2016). We have recently performed studies on the aortic root by building simulation models with or without fluid-structure interactions (Pan et al., 2014, 2015; Qiao et al., 2014; Li et al., 2019). The above-mentioned studies identified the factors of $\mathrm{H}_{\mathrm{V}}$ and effective height $(\mathrm{EH})$ as important parameters to determine the acute and long-term functions of repaired aortic valves. However, the valve repair procedure has not yet resulted in good functional stability, which indicates that this pathologic entity requires a specific approach. Thus, determining the suitable $\mathrm{H}_{\mathrm{V}}$ and reconstructing the aortic valve to adapt to the dilatation of the root at the time of the initial repair can have important prognostic implications for repair durability, which can achieve the best stabilization effect.

In this study, 20 groups of finite element models were established to simulate the process of valve opening and closing by numerical simulation. Then, the maximum stress, leaflet contact pressure, and effective orifice area (GOA) in different models were compared to evaluate valve performance. Therefore, the feasible range of the valve size in the case of continuous dilatation of the aortic root can be obtained. The obtained data might serve as basis for decision making in aortic valve repair procedures.

\section{METHODS AND MATERIALS}

\section{Three-Dimensional Geometric Modeling}

The three-dimensional geometry of a base aortic valve was constructed using geometric constraints and modeling dimensions retrieved from literature and the clinical surgical guidance as the reference model (aortic annulus diameter, $\mathrm{D}_{\mathrm{AA}}=26 \mathrm{~mm}$; sinotubular junction diameters, $\mathrm{D}_{\mathrm{STJ}}=26 \mathrm{~mm}$; sinus diameter, $\mathrm{D}_{\mathrm{S}}=40 \mathrm{~mm}$, valve height, $\mathrm{H}_{\mathrm{V}}=14 \mathrm{~mm}$; sinus height, $\mathrm{H}_{\mathrm{S}}=16 \mathrm{~mm}$ ). The left and right coronary arteries connected with the aortic sinus were removed (Figure 1) (Labrosse et al., 2011; Pan et al., 2015; Hou et al., 2019). On this basis, we optimized the geometrical structure of the leaflet and aortic sinus, and proposed a new model of the aortic root closely similar to its physiological structure.

First, the contour of the aortic sinus and leaflet was constructed using a series of arcs with different heights implemented in the SolidWorks 2015 software (Solidworks, Waltham, MA, United States), and the model with the geometric characteristics of the aortic sinus and leaflet was obtained by filling the curved surfaces. In order to conveniently constrain the variables in this study, three aortic sinuses and leaflets were assumed to be uniform and symmetrical, and used to obtain the shell structure of the aorta by putting them in an array with the center axis of the valve annulus as the rotation axis with an interval of $120^{\circ}$ (Figure 2B). Then, in order to ensure full blood flow in the aortic root and restore the real structure of 


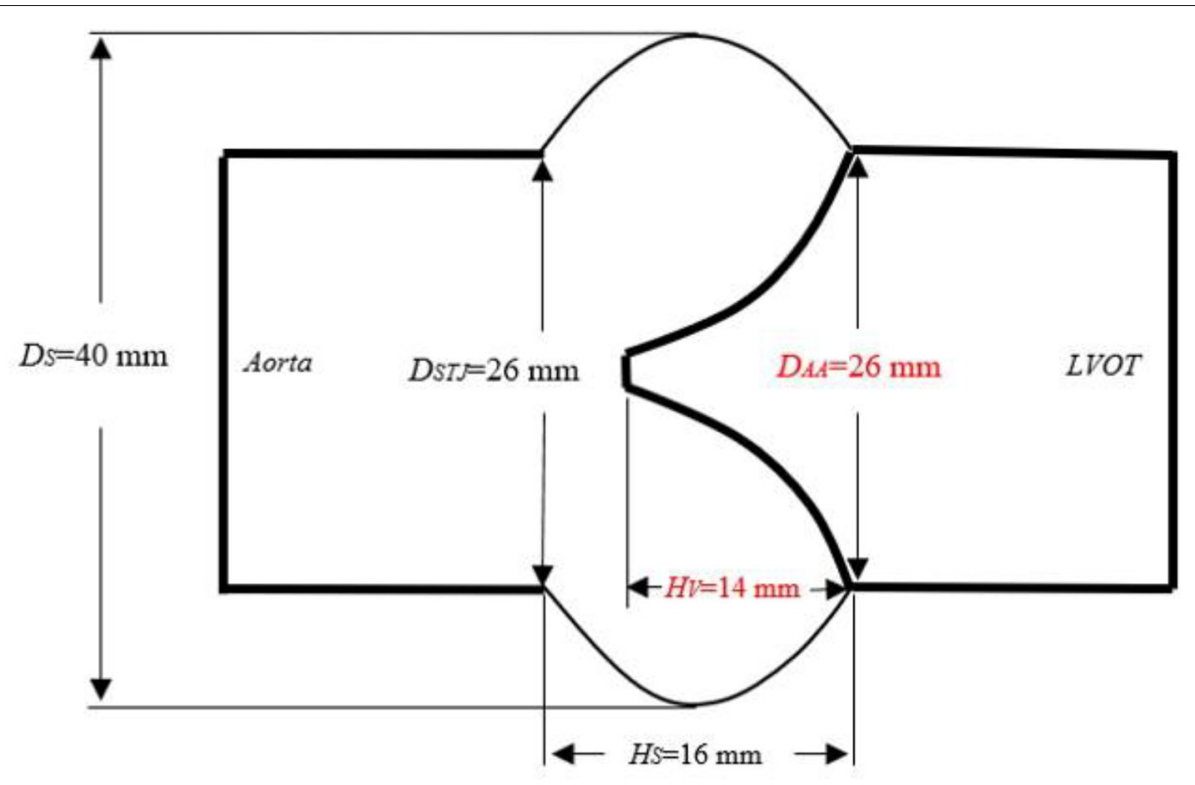

FIGURE 1 | Schematic diagram of the aortic root size. $D_{A A}$, aortic annulus diameter; $H_{V}$, valve height; $L_{H}$, valve length; $D_{S T J}$, diameter of the sinotubular junction; $\mathrm{H}_{\mathrm{S}}$, sinus height; $\mathrm{D}_{\mathrm{S}}$, sinus diameter; LVOT, left ventricular outflow tract.

the model to the greatest extent, two straight-tube extensions (length: $20 \mathrm{~mm}$ ) were added to the inlet (ventricular extension) and outlet sections (aortic extension) of the aortic root to enhance computational stability (Figure 2A) (Cao and Sucosky, 2015; Pan et al., 2015; Li et al., 2019). In all the models, the reference state consisted of the valve in a partially-open configuration (i.e., in transition between the coapted state and the opening state) and was constructed by leaving a small gap $(\mathrm{Lg}=1.5 \mathrm{~mm})$ between the free edge ( $\mathrm{Lf}=25 \mathrm{~mm}$ ) of the leaflets (Figure 2C) (Cao et al., 2016). The dimensions of the aortic valve are reported in Figure 2D (Labrosse et al., 2010; Li et al., 2019).

Based on the reference model, valve height was maintained unchanged, and aortic annulus diameter was increased first. $\mathrm{D}_{\mathrm{AA}}$ was separately set to $26,27,28,29$, and $30 \mathrm{~mm}$ to simulate the dilatation of the aortic root. Then, $\mathrm{H}_{\mathrm{V}}$ was separately set to $13.5,14,14.5$, and $15 \mathrm{~mm}$ to simulate aortic valve alterations in surgery. During the establishment of the models, other parameters remained unchanged, and $\mathrm{H}_{\mathrm{V}}$ and $\mathrm{D}_{\mathrm{AA}}$ were modified to simulate the movement and mechanics of the aortic valve under aortic root dilatation conditions. Overall, a total of 20 parameterized 3D finite element models of the aortic root were constructed.

\section{Meshing Generation and Element Settings}

All the three-dimensional models were imported into the HyperMesh 13.0 software (HyperMesh, Altair, United States) to accomplish mainly mesh generation, which included the definition of the nodes at the upper and lower ends of the aortic root and elements in different parts of the root (three leaflets, the vessel wall of the aorta, the sinus and the left ventricle outflow tract). We referred to the mesh elements that are commonly used in the existing finite element analysis of the aortic valve (Oomen et al., 2018), such that the aortic root model was divided into shell elements. The model consisted of two parts: the aortic valve and aortic wall. The shape of the artery wall was regular, which was divided into rectangular mesh according to mesh dependence analysis. Since this study mainly focused on simulation results of the sutured and free edges of the aortic valve, the meshes of these parts were all divided into neat triangular elements in order to smooth the deformed surface and determine the stress-strain relationship of the aortic valve as accurate as possible.

A mesh sensitivity analysis was performed based on the maximum stress value over the aortic valve to determine an appropriate mesh density for the model and ensure a numerical convergence. This analysis was conducted on the initial geometric model. Refining the mesh (e.g., with an element size a total of $0.4 \mathrm{~mm}$ and consisted of 7,022 elements) did not affect the stress by $<5 \%$, which was considered sufficiently resolved to capture the valvular dynamics. Therefore, this structure mesh was employed in the remaining simulations. Figure 3 shows the mesh of the overall model and the leaflets. Finally, the model was imported into the finite element software to perform varied operations, such as constraints, loading, and parameter settings.

\section{Numerical Simulation of the Aortic Root Material Properties}

In this study, the material properties and boundary conditions were set using the finite element software ADIAN 9.3 (ADINA, Watertown, MA, United States), and the numerical simulations of the structural mechanics were completed. The shell element was selected as the element type of the valve and vessel wall. Physiologically speaking, the aortic valve tissue exhibits an 


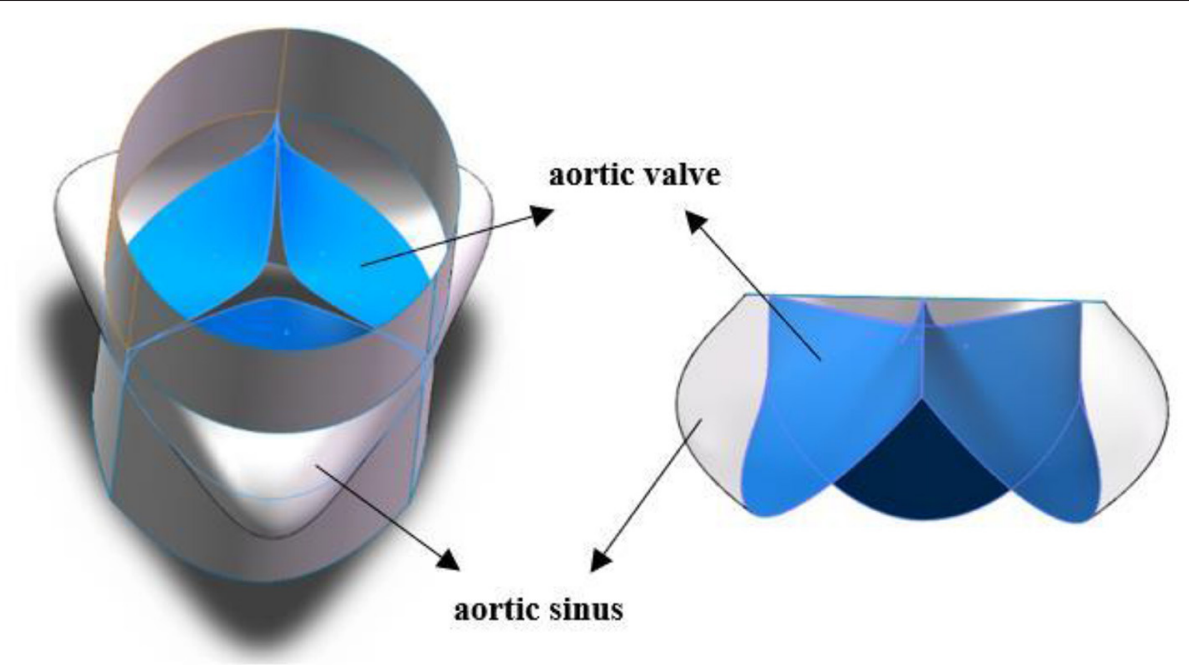

$\mathbf{A}$

B
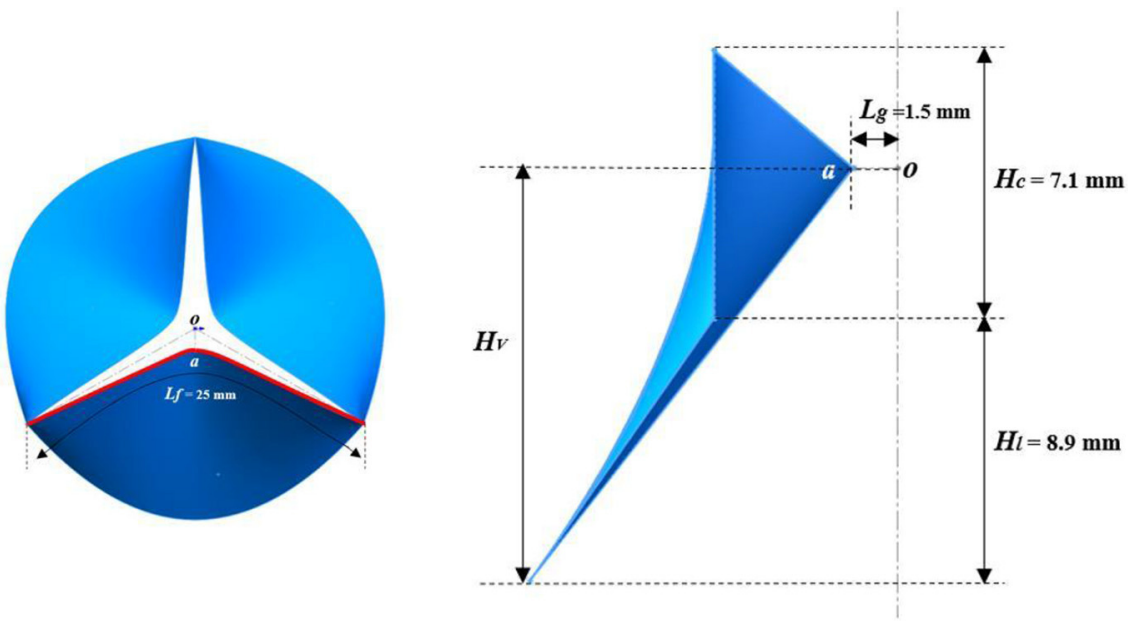

C

D

FIGURE 2 | Schematic diagram of the 3D geometry models. (A) 3D structure model of the aortic root; (B) structure of the aortic sinus and leaflet; (C) structural feature of the aortic valve; (D) dimensional characteristics of leaflet. $H_{v}$, valve height; $H_{l}$, leaflet height; $H_{c}$, commissure height; $L_{g}$, gap length; $L_{f}$, free-edge length.

obvious fiber arrangement (Feng et al., 2020), which belongs to a hyperelastic and anisotropic material. In fact, during systole, valve leaflets typically experience strains below $10 \%$ (Weinberg and Mofrad, 2007; Cao and Sucosky, 2017) and essentially behave as an isotropic material (Billiar and Sacks, 2000). The progressive locking of the collagen fibers increases material stiffness along the circumferential direction during diastole, and the valve is mostly or fully closed during this phase, which results in negligible leaflet stress levels. Therefore, the anisotropy of the leaflet material could potentially alter leaflet curvature during coaptation; it is expected to have a slow impact on regional leaflet stress. Moreover, the pressure gradient between the ventricle and the aorta is in the range of $0-14 \mathrm{kPa}$, and the stress-strain relationship of the valve leaflet is linear in this range. Therefore, the isotropic and linear elastic material properties matching the element type were assumed in the present models so as 


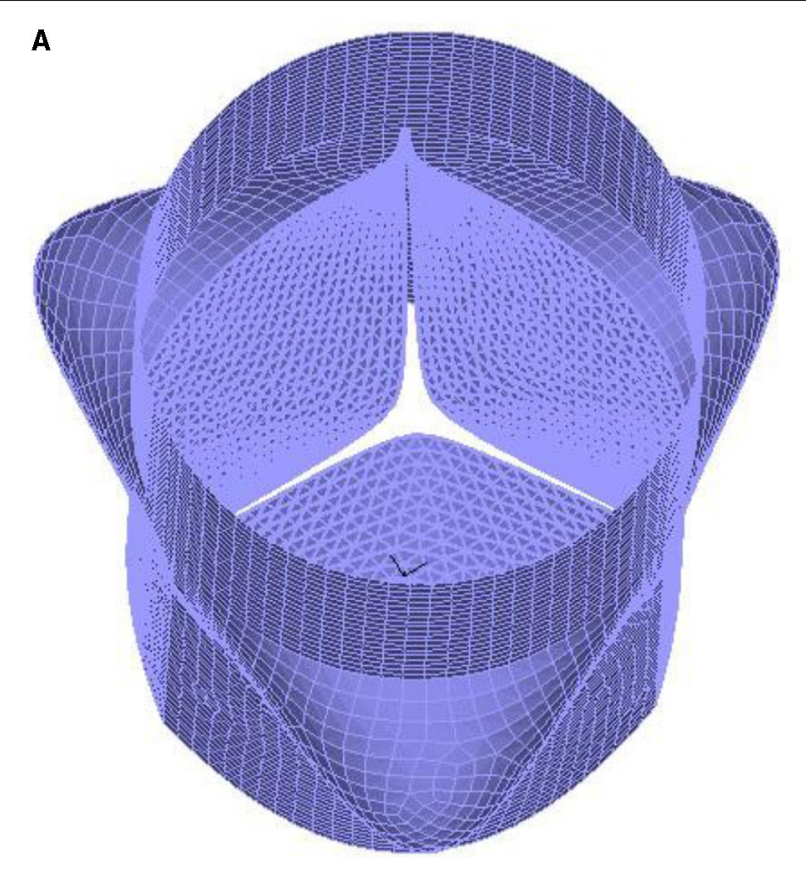

B

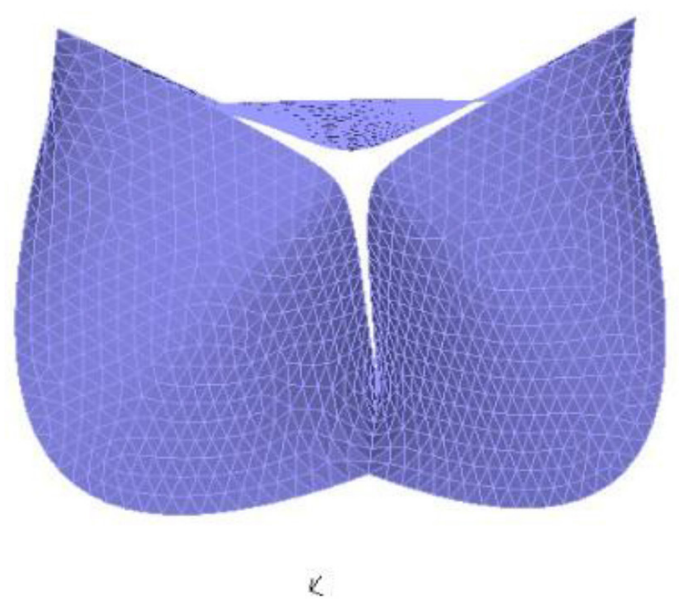

FIGURE 3 | The mesh of the aortic root. (A) the whole model; (B) the leaflets.

to simplify the computation and improve the feasibility of the analysis (Auricchio et al., 2014; Qiao et al., 2014; Hammer et al., 2016; Marom et al., 2016). This assumption is feasible to simulate the coaptation and avoid the problem of excessive distortion of the mesh during the contact process of the leaflets. Young's modulus of 1 and $2 \mathrm{MPa}$, densities of 1,100 and $2,000 \mathrm{~kg} / \mathrm{m}^{3}$, and thicknesses of 0.3 and $0.6 \mathrm{~mm}$ were used for the valve and the rest of the aortic root, respectively (Marom et al., 2012; Rim et al., 2014; Hou et al., 2019). Poisson's ratio used was 0.45 (Katayama et al., 2008; Pan et al., 2015).

\section{Boundary Conditions}

First, the nodes at the aortic outlet and ventricular outflow were fixed in all directions, with zero degrees of freedom to prevent deflection. Then, time-dependent physiological pressure conditions (Figure 4) were applied to the aorta, valve, and left ventricle. The difference between the pressure values on the side of the left ventricle and side of the aorta represented the transvalvular pressure gradient, which drives the aortic valve to move periodically (Pan et al., 2015; Li et al., 2019). In the simulation, two complete continuous pressure differences were loaded on the aortic valve. Finally, since the analysis started from the unpressurized geometry, a solution phase of $0-0.2 \mathrm{~s}$ before the systole was added to the simulation for the transition of the model from the zero-stress state to normal physiological pressure. Thus, the initial state of the model was consistent with the loading conditions at the end of the diastole, and calculation accuracy was improved (Labrosse et al., 2011; Pan et al., 2015).

\section{Computational Method and Control Parameter}

In the ADINA 9.3 (ADINA, Watertown, MA, United States) software, the implicit dynamics method based on displacement parameters was chosen to solve the partial differential equations of the structural analysis. The iteration method used was Modified Newton. In practice, in energy dissipation, the vibration response of the structure would gradually weaken without external force. The complex energy dissipation mechanism is expressed by damping, so we set the damping coefficient of the aortic valve to 0.15 to promote convergence (Weltert et al., 2013). When the aortic valve is closed, the three leaflets contact each other, and the contact type is defined as the friction contact; we set the friction coefficient to 0.01 . The time step was set to 0.001 or 0.0001 in phases where the pressure load changed slowly or drastically, respectively.

All the models were calculated using the finite element software ADINA9.3. Since periodic dependence exists in finite element analysis, the research group carried out a study on periodic issues by gradually increasing the number of cycles. The results showed that two cycles already had a good convergence effect (Pan et al., 2015). The effect of increasing the cycle did not change after two cycles, and the error of the adjacent cycles was $<5 \%$. Hence, we selected two cardiac cycles to obtain convergence results to save computation time.

\section{RESULTS}

We simulated the dynamics of the aortic root over two cardiac cycles. During the period of $0-0.2 \mathrm{~s}$, the pressure was prescribed as an initial pressurization phase. Then, the period of $0.2-0.4 \mathrm{~s}$ included rapid ejection of blood from the left ventricle into the aorta, because the left ventricular pressure exceeded the pressure within the aorta, and the aortic valve opened driven by the 


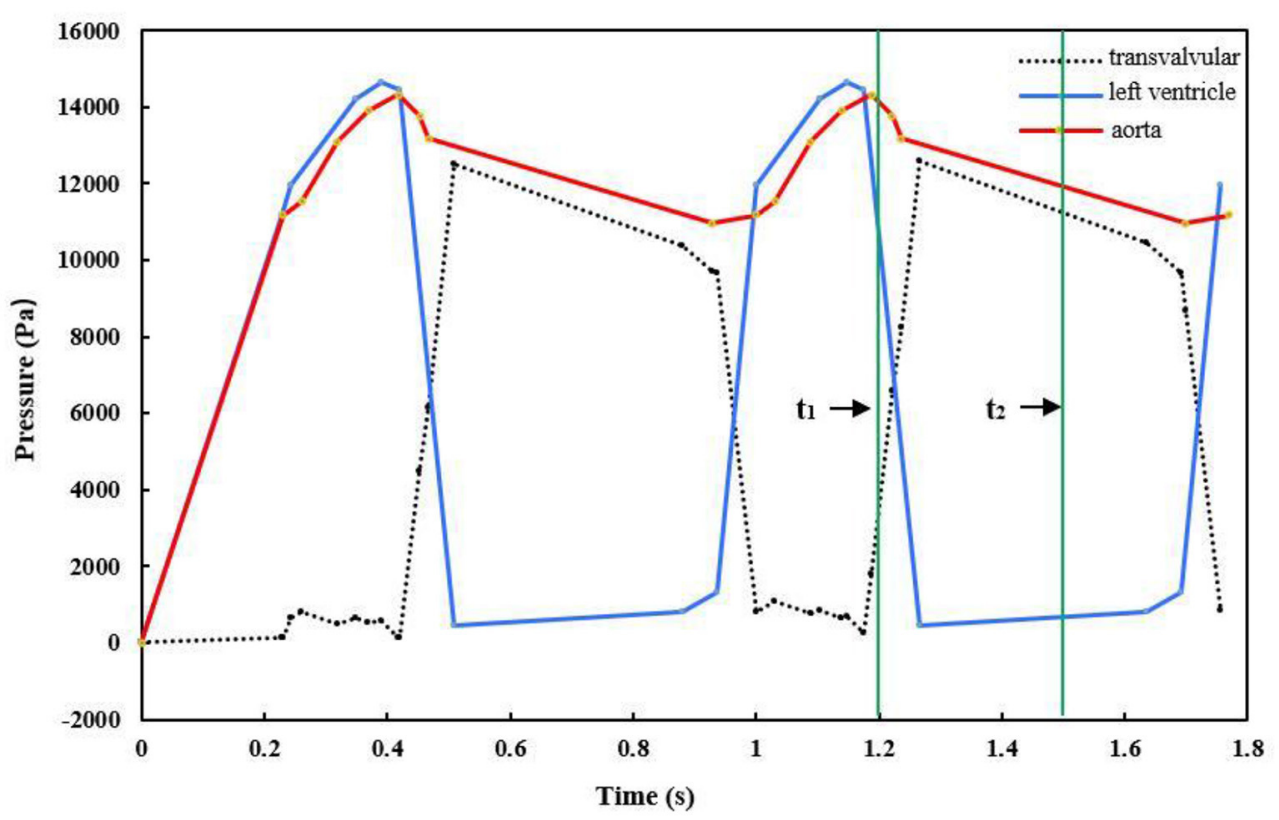

FIGURE 4 | Pressure waves exerted on the aorta, left ventricle, and leaflets (t1 $=1.2 \mathrm{~s}, \mathrm{t} 2=1.5 \mathrm{~s})$.

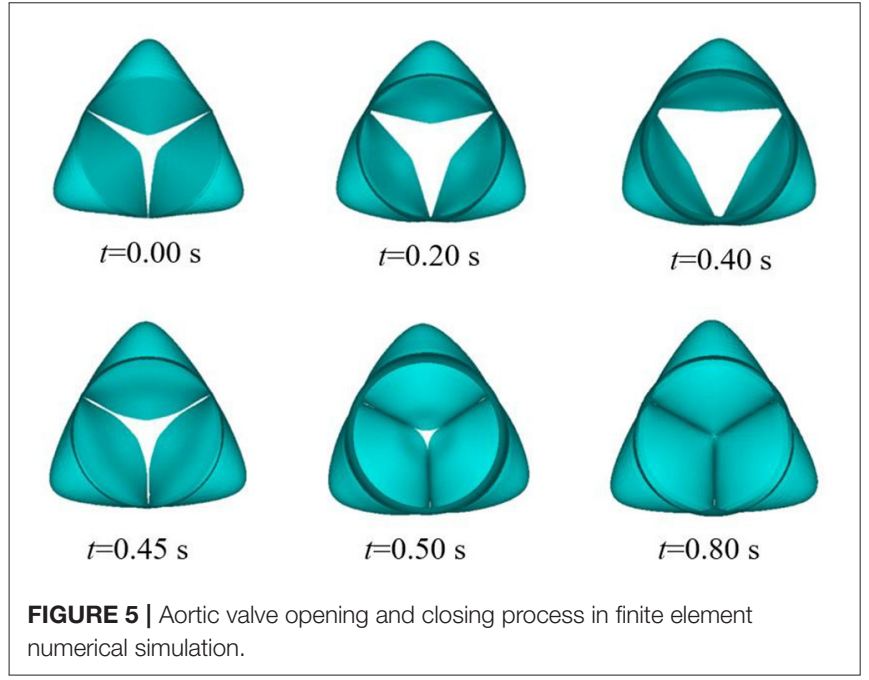

transvalvular pressure. During the period of $0.4-0.7 \mathrm{~s}$, the left ventricular pressure began to decrease to a certain value, and the aortic pressure forced the aortic valve to close. The aortic valve remained closed for a period of time, then the next cardiac cycle began. Figure 5 shows the movement of the aortic valve during a cardiac cycle in the reference model. The calculation results from the second cardiac cycle were used for the analysis. The maximum GOA of the valve was extracted at time $\mathrm{t}_{1}=1.2 \mathrm{~s}$ when the aortic valve was fully opened, while the values of the maximum stress and leaflet contact force were obtained at time $\mathrm{t}_{2}=1.5 \mathrm{~s}$ when the valve was completely closed. In the following,
TABLE 1 | Parameter values of the model with a valve height of $13.5 \mathrm{~mm}$.

\begin{tabular}{|c|c|c|c|c|c|}
\hline Parameter/D $\mathrm{AA}(\mathrm{mm})$ & 26 & 27 & 28 & 29 & 30 \\
\hline Maximum stress (kPa) & 676 & 711 & 788 & 859 & 911 \\
\hline Contact force (N) & 6.32 & 4.6 & 3.11 & 1.56 & 0.53 \\
\hline $\mathrm{GOA}\left(\mathrm{mm}^{2}\right)$ & 200.10 & 211.33 & 216.05 & 220.10 & 230.67 \\
\hline
\end{tabular}

we present the numerical simulation results according to the four cases of the valve height.

\section{Setting $\mathrm{H}_{\mathrm{V}}$ to $13.5 \mathrm{~mm}$}

The mechanical parameters of the aortic valve with an HV of $13.5 \mathrm{~mm}$ are shown in Table $\mathbf{1}$. The maximum stress value appeared to increase as the $\mathrm{D}_{\mathrm{AA}}$ increased. The contact force of the leaflets presented a decreasing trend, while the GOA of the aortic valve presented an increasing trend when the parameter $\mathrm{D}_{\mathrm{AA}}$ increased from 26 to $30 \mathrm{~mm}$.

The geometric orifice area and the maximum stress distribution of the models with a valve height of $13.5 \mathrm{~mm}$ and aortic annulus diameter of $26-30 \mathrm{~mm}$ are shown in Figure 6. It can be found that the maximum stress was in accordance with what was reported in previous studies (Labrosse et al., 2011; Marom et al., 2013) when the $\mathrm{D}_{\mathrm{AA}}$ was 26,27 , or $28 \mathrm{~mm}$. Meanwhile, the contact force of the leaflets was close to the results of $5.43 \mathrm{~N}$ in the study of Qiao et al. (2014). The orifice area of the valve was $>200 \mathrm{~mm}^{2}$, which can meet the requirement of the clinical standard. When the $\mathrm{D}_{\mathrm{AA}}$ was 29 or $30 \mathrm{~mm}$, the GOA was within the normal range, and the contact force was slightly smaller. Larger stress occurred at the junction of the 


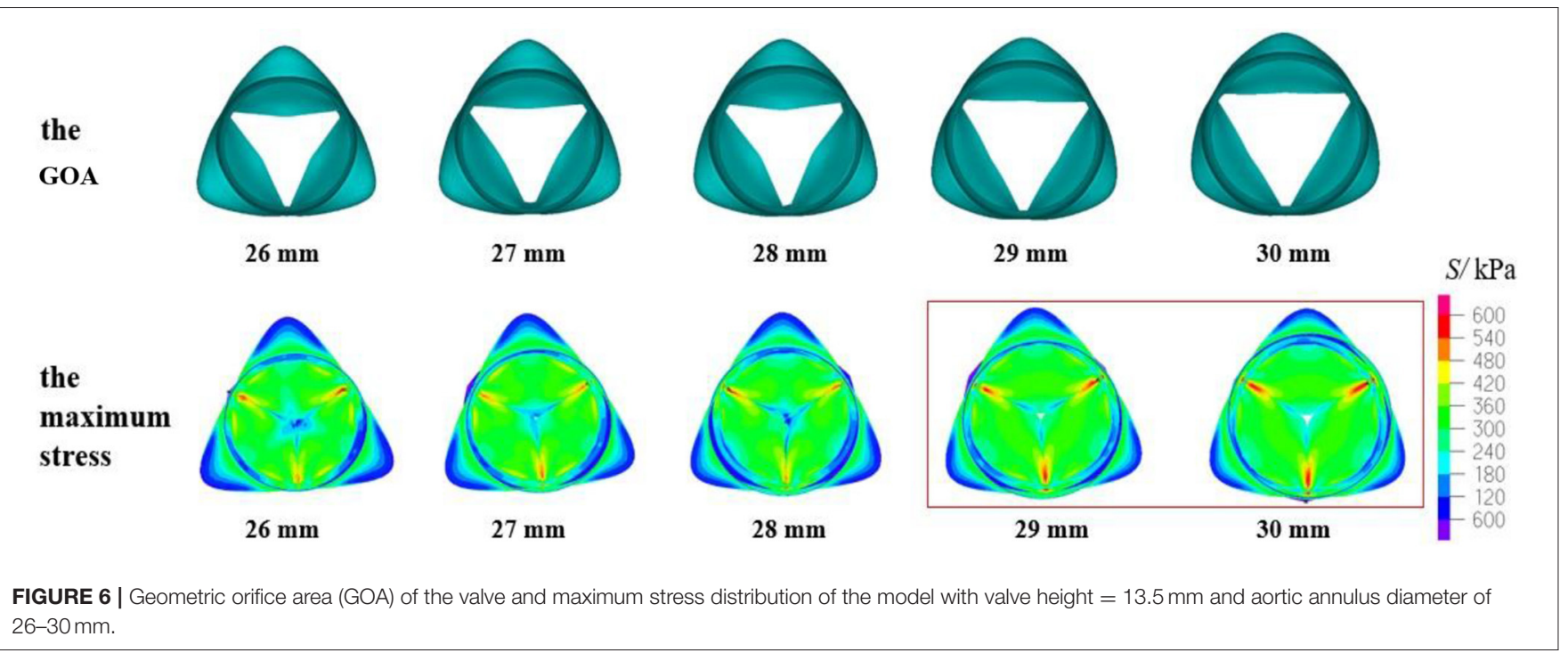

TABLE 2 | Parameter values of the model with a valve height of $14 \mathrm{~mm}$.

\begin{tabular}{|c|c|c|c|c|c|}
\hline Parameter/D $\mathrm{D}_{\mathrm{AA}}(\mathrm{mm})$ & 26 & 27 & 28 & 29 & 30 \\
\hline Maximum stress (kPa) & 629 & 757 & 878 & 864 & 893 \\
\hline Contact force (N) & 7.09 & 5.52 & 4.22 & 3.47 & 3.10 \\
\hline $\mathrm{GOA}\left(\mathrm{mm}^{2}\right)$ & 209.10 & 209.75 & 210.11 & 216.34 & 230.67 \\
\hline
\end{tabular}

leaflets and sinus, and there were several incomplete closures of the leaflet joints, while the maximum stress and contact force were relatively small. The reason may be the large insufficiency of the valve at $1.5 \mathrm{~s}$ of the second cardiac cycle.

\section{Setting $\mathrm{H}_{\mathrm{V}}$ to $14 \mathrm{~mm}$}

The mechanical parameters of the aortic valve with a valve height of $14 \mathrm{~mm}$ are shown in Table 2. The GOA of the aortic valve showed an increasing trend, while the contact force of the leaflets tended to decrease as the $\mathrm{D}_{\mathrm{AA}}$ increased from 26 to $30 \mathrm{~mm}$.

The geometric orifice area and maximum stress distribution of the models with a valve height of $14 \mathrm{~mm}$ and aortic annulus diameter of $26-30 \mathrm{~mm}$ are shown in Figure 7. When the $\mathrm{D}_{\mathrm{AA}}$ was 26,27 , or $28 \mathrm{~mm}$, the maximum stress and the contact force of the leaflets were similar to the previously reported simulation results (Howard et al., 2003; Marom et al., 2013; Weltert et al., 2013). The GOA met the clinical standard. When the $\mathrm{D}_{\mathrm{AA}}$ was 29 or $30 \mathrm{~mm}$, the GOA was within the normal range, but the maximum stress values were $>800 \mathrm{kPa}$ and greater stress also occurred at the junction of the leaflets and sinus. On the other hand, the contact force was relatively small, similar to the model with an $\mathrm{H}_{\mathrm{V}}$ of $13.5 \mathrm{~mm}$, which represents the major risk factor for AI.

\section{Setting $H_{V}$ to $14.5 \mathrm{~mm}$}

The mechanical parameters of the aortic valve with a valve height of $14.5 \mathrm{~mm}$ are shown in Table 3. When the $\mathrm{H}_{\mathrm{V}}$ was $14.5 \mathrm{~mm}$, the three evaluation parameters affecting the opening and closing performance of the aortic valve were within a reasonable range, and the aortic valve of all the models could achieve complete closure and opening. Figure 8 shows the GOA and maximum stress distribution of the models with an $\mathrm{H}_{\mathrm{V}}$ of $14.5 \mathrm{~mm}$ and $\mathrm{D}_{\mathrm{AA}}$ of 26-30 mm. A comparison of the three parameters between the models with an $\mathrm{H}_{\mathrm{V}}$ of 14 and $14.5 \mathrm{~mm}$, respectively, is shown in Figure 9.

All the models with a valve height of 14 and $14.5 \mathrm{~mm}$ had complete closure and normal movement of the leaflets. The models with an $\mathrm{H}_{\mathrm{V}}$ of $14.5 \mathrm{~mm}$ had smaller stress, larger GOA, and little change in the contact force compared with the models with an $\mathrm{H}_{\mathrm{V}}$ of $14 \mathrm{~mm}$ (Figure 9). Thus, small maximum stress, reasonable leaflet contact force, and GOA have the smallest influence on the opening and closing performance of the aortic valve and are most consistent with the normal physiological state of the human body.

\section{Setting $H_{V}$ to $15 \mathrm{~mm}$}

The mechanical parameters of the aortic valve with a valve height of $15 \mathrm{~mm}$ are shown in Table 4 . Under the five values of $\mathrm{D}_{\mathrm{AA}}$, the maximum stress and the leaflet contact force were within the normal range. However, according to the values of the GOA and the contact force (shown in Figure 10), the GOA was lower than the standard value, and the contact forces were greater than the other models when the $\mathrm{D}_{\mathrm{AA}}$ was 26 or $27 \mathrm{~mm}$. When the $\mathrm{D}_{\mathrm{AA}}$ was 28,29 , or $30 \mathrm{~mm}$, an $\mathrm{H}_{\mathrm{V}}$ value of $15 \mathrm{~mm}$ could be well adapted.

\section{DISCUSSION}

Aortic valve repair is an attractive alternative to prosthetic valve replacement in young patients with aortic insufficiency (Navarra et al., 2013; Emmanuel et al., 2016). The repair procedure has advantages of low occurrence rates of valve-related events and 


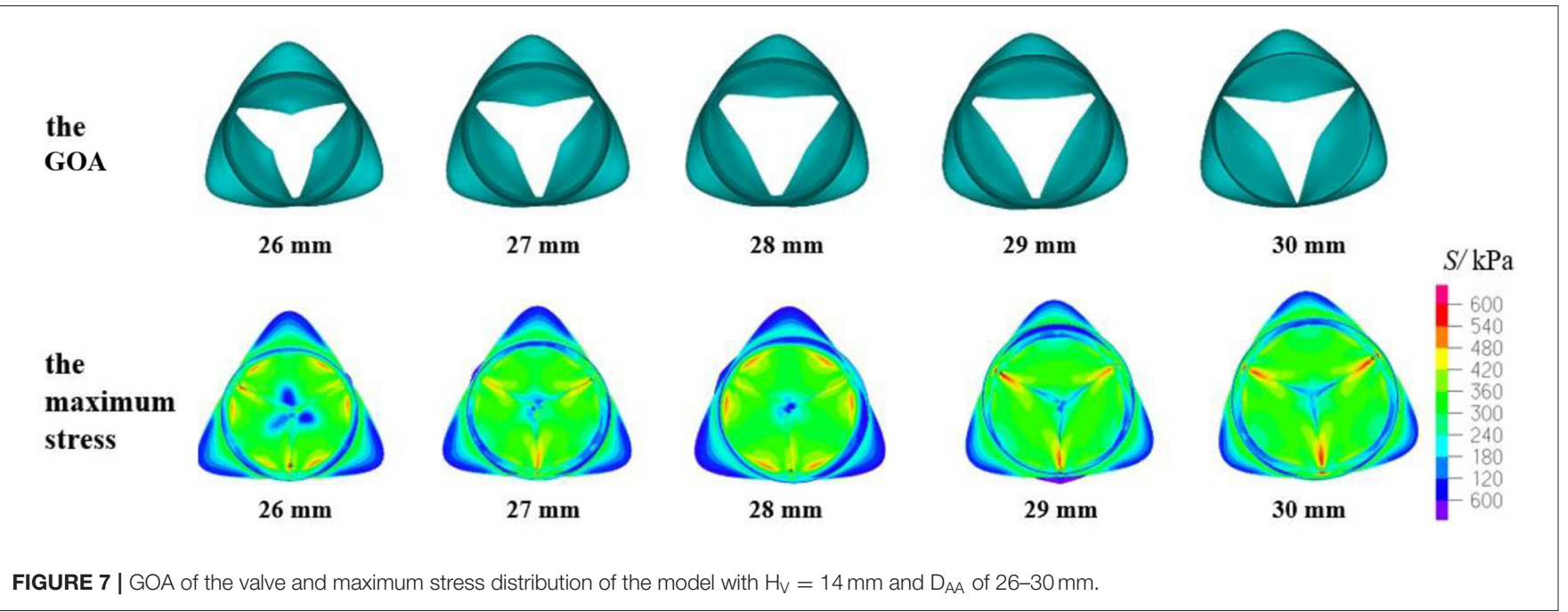

TABLE 3 | Parameter values of the model with a valve height of $14.5 \mathrm{~mm}$.

\begin{tabular}{|c|c|c|c|c|c|}
\hline Parameter/D $\mathrm{D}_{\mathrm{AA}}(\mathrm{mm})$ & 26 & 27 & 28 & 29 & 30 \\
\hline Maximum stress (kPa) & 639 & 701 & 576 & 741 & 793 \\
\hline Contact force (N) & 8.46 & 7.03 & 7.55 & 7.71 & 6.66 \\
\hline $\mathrm{GOA}\left(\mathrm{mm}^{2}\right)$ & 215.30 & 234.39 & 207.17 & 259.90 & 251.33 \\
\hline
\end{tabular}

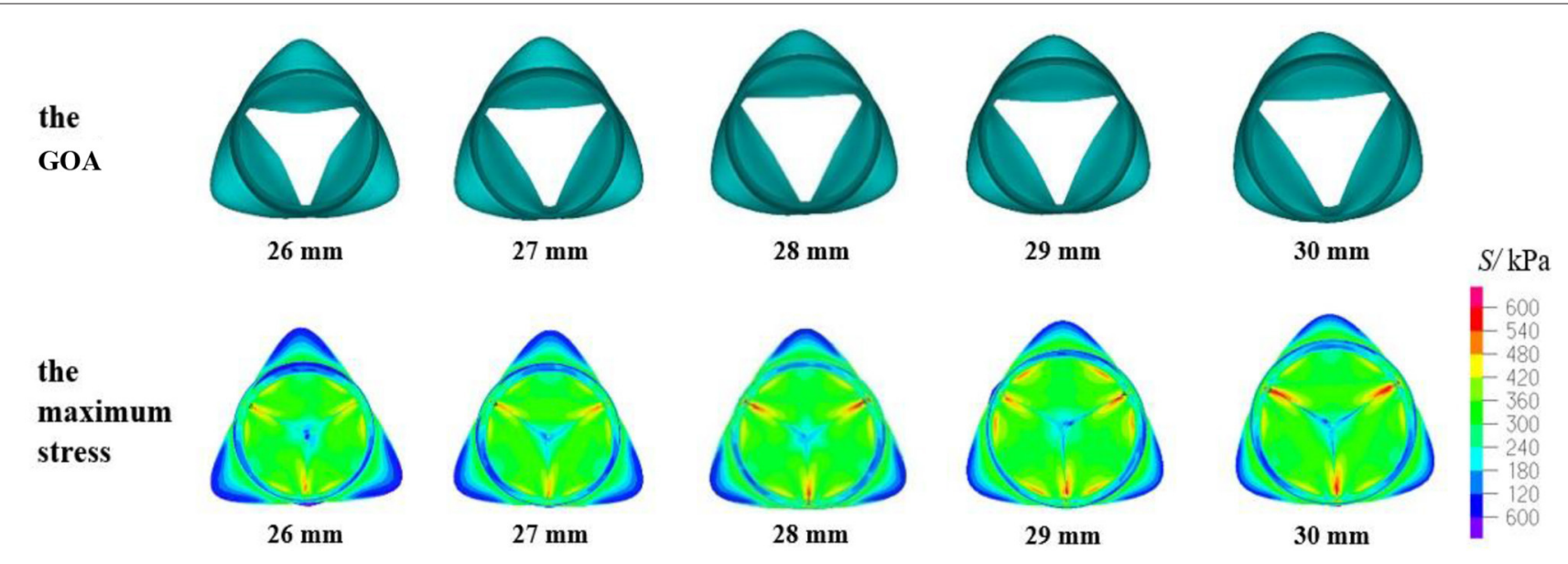

FIGURE 8 | GOA of the valve and maximum stress distribution of the model with $\mathrm{H}_{V}=14.5 \mathrm{~mm}$ and $\mathrm{D}_{\mathrm{AA}}$ of $26-30 \mathrm{~mm}$.

there is no need for lifelong anticoagulation therapy. However, the repaired valve may develop functional failure over time. Durability limitations become apparent by the end of the first postoperative decade, mainly because of progressive dilatation of the aortic root.

The three-dimensional geometry of a base aortic root was reconstructed using the geometric constraints and modeling dimensions suggested by Labrosse et al. (2011). Then, the diameters of the aortic annulus and the height of the aortic valve were modified to create 20 geometric models with different dimensions. In this study, we used the structural mechanics simulation method to simulate the process of aortic valve movement and simulated the dynamics of the aortic root over two cardiac cycles. Next, we used the data from the last cardiac cycle for the analysis. The performance of the aortic valve was quantified in terms of maximum stress, GOA, and leaflet contact force.

\section{Analysis of Different Models Affecting Maximum Stress}

The maximum stress value is an important index to evaluate the mechanical performance of the valve. From a physiological aspect, excessive stress may tear the valve or accelerate 


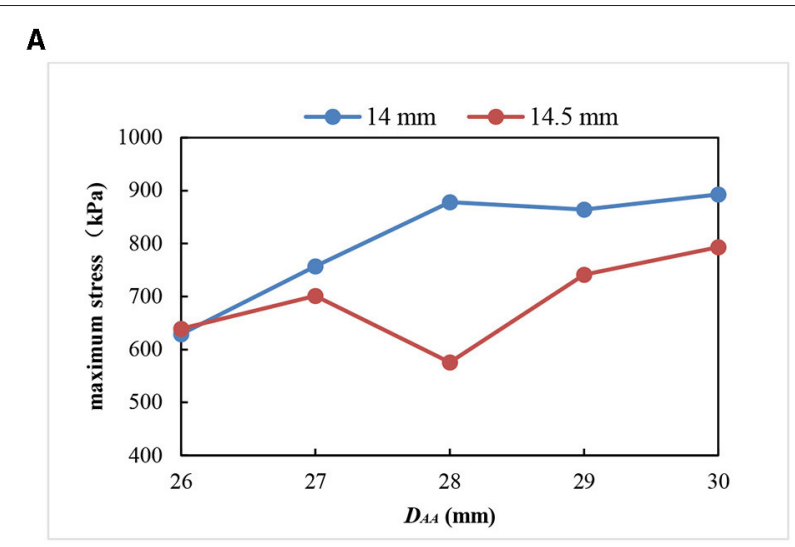

B

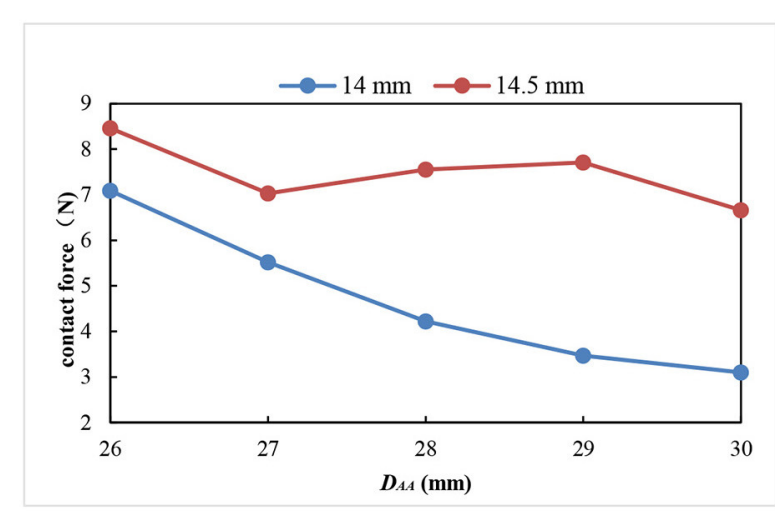

C

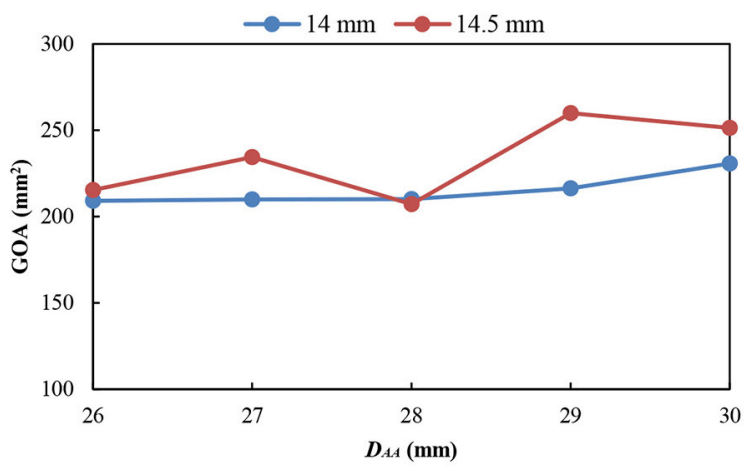

FIGURE 9 | Comparison of three parameters between the models with an $\mathrm{H}_{V}$ of 14 and $14.5 \mathrm{~mm}$. (A) Maximum stress; (B) contact force; (C) geometric orifice area.

the calcification process of the aortic valve because of the accumulation of inactive cells (Cao and Sucosky, 2017; Jia et al., 2017), thereby further aggravating aortic stenosis. Therefore, the value and distribution of the maximum stress during valve
TABLE 4 | Parameter values of the model with a valve height of $15 \mathrm{~mm}$.

\begin{tabular}{|c|c|c|c|c|c|}
\hline Parameter/D $\mathrm{AA}(\mathrm{mm})$ & 26 & 27 & 28 & 29 & 30 \\
\hline Maximum stress (kPa) & 677 & 653 & 672 & 603 & 569 \\
\hline Contact force (N) & 9.01 & 8.56 & 7.34 & 7.01 & 6.32 \\
\hline $\mathrm{GOA}\left(\mathrm{mm}^{2}\right)$ & 177.33 & 198.54 & 201.33 & 215.23 & 220.74 \\
\hline
\end{tabular}

closure are of great importance to clinicians and researchers. The results of this study showed that the maximum stress presented a trend of weakly decreasing first and then sharply increasing in the cardiac cycle, bearing the highest stress during diastole. A previous study (Labrosse et al., 2010) has shown the maximum stress value for the aortic valve to be $600-750 \mathrm{kPa}$ during the diastolic period. Qiao et al. used 3D structural models to study the influence of the sinotubular junction and sinus diameter on the valve. They reported the calculated maximum stress range of the model with normal motion in numerical simulation to be 567-601 kPa (Qiao et al., 2014). Marom studied the effect of $D_{A A}$ on the valve and calculated the maximum stress to be $800 \mathrm{kPa}$ (Marom et al., 2013). The results in this study showed that the maximum stress was generally distributed at the junction of the aortic valve and sinus. All the AI models had maximum stress values $>800 \mathrm{kPa}$, and the degree of insufficiency became more serious as the stress increased. Therefore, the smaller the stress on the valve leaflet, the more conducive the efforts to maintain the long-term effectivity of the aortic valve under the premise of ensuring its normal function.

\section{Analysis of Different Models Affecting the Leaflet Contact Force}

Leaflet contact force refers to the interaction force between leaflets. It could be indicative of the holding strength between leaflets during closing phases of the cardiac cycle (Marom et al., 2013; Pan et al., 2015). Dynamic leaflet contact force data generated during the simulation were saved to a file to perform further processing using the MATLAB environment. This was done to determine the surface area of coaptation at the time of maximum downward movement of the leaflets during diastole (Labrosse et al., 2011).

The simulation results show that just after initial leaflet separation, the pressure was ramped back down to $0 \mathrm{kPa}$ with the contact management turned on, which allowed the leaflets to contact each other in a realistic fashion. The pressure was then raised to $13 \mathrm{kPa}$. The contact force applied on the coaptation area was not a parameter that exhibited large variations, ranging from 0.5 to $9 \mathrm{~N}$ (Tables 1-4). This pressure was calculated during diastole at the time when the pressure differential between the aortic and ventricular sides was $13 \mathrm{kPa}$. It makes intuitive sense that the higher the leaflet contact force, the better the valve coaptation. However, in general, this parameter does not appear to be a good predictor of coaptation quality. Excessive contact force can increase the energy consumption of leaflets, increase the risk of $\mathrm{AR}$, and reduce the durability of the aortic valve. In this study, the contact force in the model in which the valve can be normally closed was $>3 \mathrm{~N}$ during the whole cardiac cycle. This 


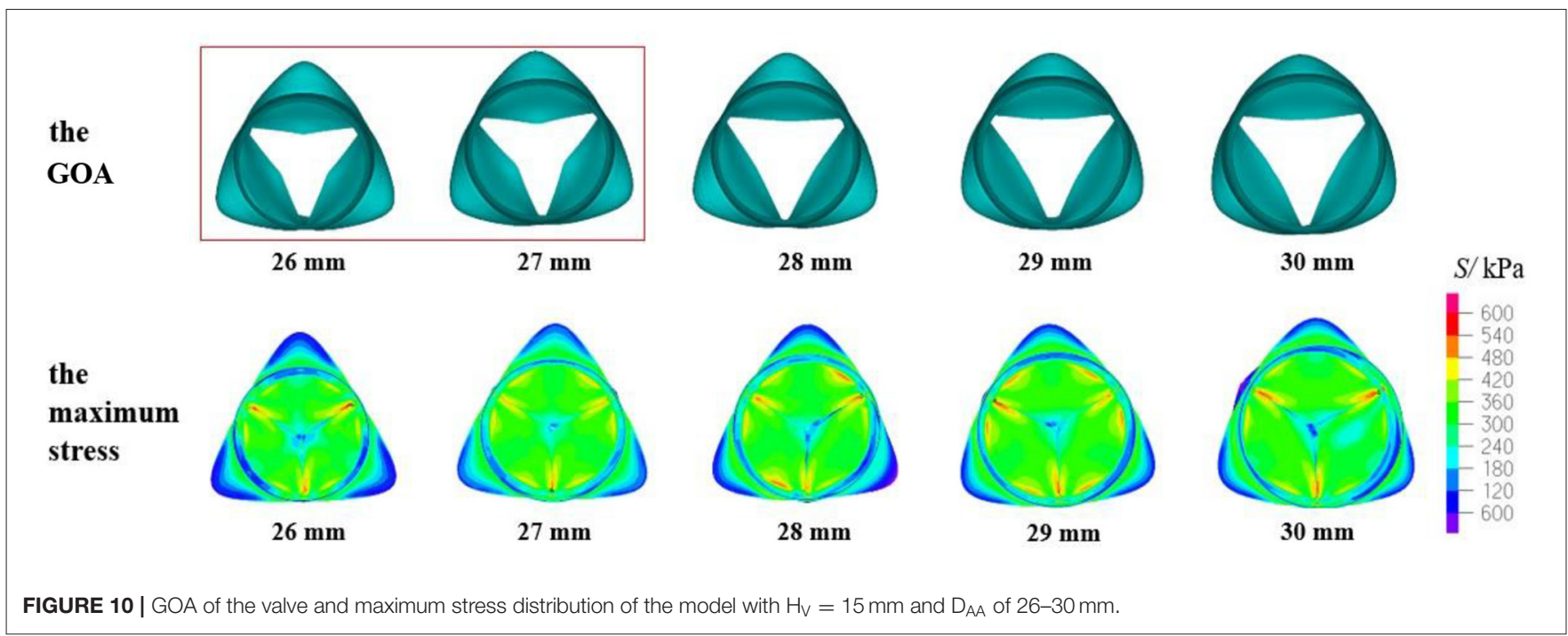

phenomenon is similar to the results previously reported by $\mathrm{Li}$ et al. (2019). In the model where the leaflet contact force was $<3 \mathrm{~N}$, the valve was incompletely closed. As a result, the contact between the leaflets may be abnormal if the contact force is too large or too small.

\section{Analysis of Different Models Affecting the GOA of Leaflet}

Geometric orifice area is the critical reference index to evaluate the opening and closing characteristics of the aortic valve, the larger the opening area, the better the opening effect of the heart valve. First, we needed to determine the moment when the GOA has the largest value, and then the post-processor of ADINA was used to take top view snapshots of the valve at that moment during the cardiac cycle by projecting the valve on the virtual valvular ring plane. Lastly, we drew the space surface of the opening area according to the projected area and calculated its surface area using the SolidWorks 15.0 software.

According to the criteria of clinical diagnosis, the aortic valve orifice area could be defined according to three states: when the geometric orifice area is $<200$ and more than $150 \mathrm{~mm}^{2}$, it is mild stenosis; when the geometric orifice area is $<150$ and more than $100 \mathrm{~mm}^{2}$, it is moderate stenosis; when the geometric orifice area is $<100 \mathrm{~mm}^{2}$, it is severe stenosis. Therefore, the clinical standard value for normal GOA is $>200 \mathrm{~mm}^{2}$ (Garcia et al., 2011). The aortic valve orifice area with the valve in the fully open position for the TAV is shown in Figures 6, 7, 8, 10. The open area shown in these figures is more like a triangle but not a circle. This phenomenon is related with the geometry and material of the prosthetic valves. The demonstrated scenario is similar to that reported in Labrosse et al. and others (Kim et al., 2007, 2008; Jermihov et al., 2011; Labrosse et al., 2011; Hsu et al., 2014; Gilmanov and Sotiropoulos, 2016). Previous studies (Labrosse et al., 2011; Li et al., 2019) have calculated the maximum orifice area of all aortic root models with a normal valve function to be $210 \pm 10 \mathrm{~mm}^{2}$, which compared well with in vivo data obtained from transesophageal echocardiography in
19 normal aortic valves with a maximum GOA of $270 \pm 63 \mathrm{~mm}^{2}$. This is close to the calculated results in this article.

The results of this study show that only in two models $\left(\mathrm{H}_{\mathrm{V}}=15 \mathrm{~mm}, \mathrm{D}_{\mathrm{AA}}=26,27 \mathrm{~mm}\right)$ the geometric orifice area was $<200 \mathrm{~mm}^{2}$, and that it was more than $200 \mathrm{~mm}^{2}$ in the other models, which met the standard value. However, when the GOA is $<200 \mathrm{~mm}^{2}$, the aortic valve may suffer from stenosis, which leads to the obstruction of the left ventricular blood output and an increase in the afterload. The heart will, in fact, increase its own work to improve the cardiac output to satisfy the blood supply needs of the body, which is called the Starling compensation mechanism. Therefore, from the long-term development of the disease, this will increase the burden of the heart, which may cause serious heart diseases, such as myocardial thickening, cardiac hypertrophy, and other serious heart diseases.

\section{Limitations and Future Study}

In this study, several assumptions on the model settings and materials were made to reduce complexity. The main limitations of this study include the following aspects: first, parameterized ideal models based on physiological anatomy were used instead of a patient-specific model. Although patient-specific models could actually reflect the morphology and structure of diseased valves and aortic roots, morphological differences between individuals were huge and sizes varied (Cao and Sucosky, 2015), making it difficult to quantitatively compare the differences between aortic roots. Parameterized models have been used to study the normal aortic valve function (Labrosse et al., 2011; Weltert et al., 2013; Pan et al., 2015; Halevi et al., 2016) and surgical repair techniques (Labrosse et al., 2011; Hammer et al., 2015). For these studies, the primary utility of computational methods is the ability to isolate and quantify the effect of single variables, such as size, shape, and mechanical properties of a given structure on measurable outcomes. In order to highlight the structural differences between different aortic roots and reduce the influence of other factors in the models, parameterized models were adopted in this study. Based on the initial model, 
the root dilatation and valve alterations in surgery were simulated by changing the annulus diameter and valve height, respectively, while the dimensions of other components, such as sinus diameter and valve height, remained unchanged. Among them, the three aortic sinuses and leaflets were assumed to be uniform and symmetrical, and the left and right coronary arteries were not considered in the sinus model, which was somewhat different from the physiological anatomy. The influence of the coronary artery structure on aortic valve closure function needs to be further discussed in the next study. Second, in this simulation study, the material of the aortic valve was assumed to have isotropic and linear elastic material properties to simplify the computation and improve the feasibility of the analysis and avoid the problem of excessive distortion of the mesh during the contact process of the leaflets. In fact, the aortic valve tissue exhibits an obvious fiber arrangement (Feng et al., 2020), which is a characteristic of a hyperelastic and anisotropic material. Studies have shown that isotropic and anisotropic leaflet materials are not expected to significantly affect the dynamic performance of leaflets (Hammer et al., 2016; Cao and Sucosky, 2017). However, in order to study the mechanical properties of the valve, including stress and strain on leaflets, it may be necessary to consider the anisotropic constitutive mode in future studies.

Finally, under normal physiological conditions of the human body, the aortic root is in the flow field. When the blood flow from the left ventricle acts on aortic root components, such as the leaflets and sinus, stress distribution is different in each part. However, the idealized model of the aortic root constructed in this study did not consider the influence of blood flow, and the pressure load was uniformly applied to the valve and the vessel wall. Nevertheless, in the fluid-structure interaction (FSI) simulation study (Marom et al., 2016), the movement of the commissures was found to be somewhat small $(<2 \%)$. This simplification is not expected to significantly affect the kinematics of the leaflets, and therefore should have a great influence on hemodynamic and wall shear stress (WSS) predictions. The purpose of this study was mainly to focus on the mechanical properties and structural changes of the leaflets. Some studies have pointed out that structural simulations are forced to assume spatially uniform transvalvular pressure (Haj-Ali et al., 2008; Conti et al., 2010a,b), which was found to be higher than the pressure load on the leaflets. As the leaflet tissue is soft, this pressure load is enough to deform the leaflet. The larger pressure load yields larger coaptation area, contact force, and stress in the structural model. Therefore, the use of structural models is justified for stress analysis, durability, or calcification evaluation,

\section{REFERENCES}

Aicher, D., Schneider, U., Schmied, W., Kunihara, T., Tochii, M., and Schäfers, H. J. (2012). Early results with annular support in reconstruction of the bicuspid aortic valve. J. Thorac. Cardiov. Surg. 145, S30-S34. doi: 10.1016/j.jtcvs.2012.11.059

Alkhodari, M., and Fraiwan, L. (2021). Convolutional and recurrent neural networks for the detection of valvular heart diseases in since the larger predicted stress gives a conservative estimate. In future studies, fluid-structure coupling analysis and pulsating flow experiments in vitro can be performed to obtain a more comprehensive and accurate conclusion.

\section{CONCLUSION}

This study has shown that aortic annulus diameter and valve height are important factors influencing the performance of aortic valve closure, especially with regard to valve repair operation. The results show that within the 20 designed models, the aortic valve geometry with an $\mathrm{H}_{\mathrm{V}}$ of $13.5 \mathrm{~mm}$ is suitable for the small aortic root geometry, and that best choices for $\mathrm{D}_{\mathrm{AA}}$ are 26, 27, or $28 \mathrm{~mm}$. An $\mathrm{H}_{\mathrm{V}}$ of 14 or $14.5 \mathrm{~mm}$ can well adapt to the five selected $\mathrm{D}_{\mathrm{AA}}$. Compared with the model with an $\mathrm{H}_{\mathrm{V}}$ of $14 \mathrm{~mm}$, the model with an $\mathrm{H}_{\mathrm{V}}$ of $14.5 \mathrm{~mm}$ has relatively small stress, large GOA, and little change in the contact force. The aortic valve with an $\mathrm{H}_{\mathrm{V}}$ of $15 \mathrm{~mm}$ is suitable for the larger aortic root and better choices for $\mathrm{D}_{\mathrm{AA}}$ are 28,29 , or $30 \mathrm{~mm}$. Hence, a smaller $\mathrm{H}_{V}$ is adapted to a smaller $\mathrm{D}_{\mathrm{AA}}$ and vice versa. When $\mathrm{H}_{\mathrm{V}}$ is $14.5 \mathrm{~mm}$, the mechanical performance of the valve is good and can well adapt to the dilatation of the aortic root to enhance repair durability. All these findings suggest that more attention should be paid to $\mathrm{H}_{\mathrm{V}}$ during surgical planning.

\section{DATA AVAILABILITY STATEMENT}

The original contributions presented in the study are included in the article/supplementary material, further inquiries can be directed to the corresponding author/s.

\section{AUTHOR CONTRIBUTIONS}

QH was responsible for modeling, simulation, data analysis, and article preparation. GL assisted in data analysis. HL assisted in the design of numerical simulation. NL, HoZ, ZQ, and HaZ were responsible for language modification. YP assisted in the construction of the 3D model. AQ was responsible for supervision. All authors contributed to the article and approved the submitted version.

\section{FUNDING}

This study was supported by the National Natural Science Foundation of China (Grant No: 11902011). R., et al. (2015). Reported outcome after valve-sparing aortic root replacement for aortic root aneurysm: a systematic review and meta-analysis-sciencedirect. Ann Thorac. Surg. 100, 1126-1131. doi: 10.1016/j.athoracsur.2015.05.093

Auricchio, F., Conti, M., Morganti, S., and Reali, A. (2014). Simulation of transcatheter aortic valve implantation: a patient-specific 
finite element approach. Comput.Method. Biomed. 17, 1347-1357. doi: $10.1080 / 10255842.2012 .746676$

Billiar, K. L., and Sacks, M. S. (2000). Biaxial mechanical properties of the natural and glutaraldehyde treated aortic valve cusp-Part I: experimental results. J. Biomech. Eng. 122, 23-30. doi: 10.1115/1.429624

Cao, K., Buka, M., and Sucosky, P. (2016). Three-dimensional macro-scale assessment of regional and temporal wall shear stress characteristics on aortic valve leaflets. Comput. Method. Biomech. Biomed Eng. 19, 1-11. doi: 10.1080/10255842.2015.1052419

Cao, K., and Sucosky, P. (2015). Effect of bicuspid aortic valve cusp fusion on aorta wall shear stress: preliminary computational assessment and implication for aortic dilation. World J. Cardiovasc. Dis. 05, 129-140. doi: 10.4236/wjcd.2015.56016

Cao, K., and Sucosky, P. (2017). Computational comparison of regional stress and deformation characteristics in tricuspid and bicuspid aortic valve leaflets. Int. J. Numer. Methods Biol. 33:e2798. doi: 10.1002/cnm.2798

Conti, C. A., Corte, A. D., Votta, E., Viscovo, L. D., Bancone, C., Santo, L., et al. (2010a). Biomechanical implications of the congenital bicuspid aortic valve: a finite element study of aortic root function from in vivo data. J. Thorac. Cardio. Surg. 140, 890-896. doi: 10.1016/j.jtcvs.2010.01.016

Conti, C. A., Votta, E., Corte, A. D., Viscovo, L. D., Bancone, C., Cotrufo, M., et al. (2010b). Dynamic finite element analysis of the aortic root from mriderived parameters. Med. Eng. Phys. 32, 212-221. doi: 10.1016/j.medengphy.2009.12.003

David, T. E. (2013). Surgical treatment of aortic valve disease. J. Nat. Rev. Cardiol. 10, 375-386. doi: 10.1038/nrcardio.2013.72

Emmanuel, L., Di, C. I., Ghassan, S., Stephanie, L., Nizar, K., Alain, B., et al. (2016). Long-term results of external aortic ring annuloplasty for aortic valve repair. Eur. J. Cardio. Thorac. 50, 350-360. doi: 10.1093/ejcts/ezw070

Feng, Y., Cao, Y., Wang, W., Zhang, H., Wei, L., Jia, B., et al. (2020). Computational modeling for surgical reconstruction of aortic valve by using autologous pericardium. IEEE. Access.8, 97343-97352. doi: 10.1109/ACCESS.2020.2997605

Garcia, J., Kadem, L., Larose, E., Clavel, M. A., and Pibarot, P. (2011). Comparison between cardiovascular magnetic resonance and transthoracic Doppler echocardiography for the estimation of effective orifice area in aortic stenosis. J. Cardiovasc. Magn. Reson. 13, 1-9. doi: 10.1186/1532-42 9X-13-25

Gilmanov, A., and Sotiropoulos,F. (2016). Comparative hemodynamics in an aorta with bicuspid and trileaflet valves. Theor. Comput. Fluid Dyn. 30, 67-85. doi: 10.1007/s00162-015-0364-7

Gnyaneshwar, R., Kumar, R. K., and Balakrishnan, K. R. (2002). Dynamic analysis of the aortic valve using a finite element model. Ann. Thorac. Surg. 73, 1122-1129. doi: 10.1016/S0003-4975(01)03588-3

Goldstone, A. B., Chiu, P., Baiocchi, M., Lingala, B., Patrick, W. L., Fischbein, M. P., et al. (2017). Mechanical or biologic prostheses for aortic-valve and mitral-valve replacement. New. Engl. J. Med. 377, 1847. doi: 10.1056/NEJMoa16 13792

Haj-Ali, R., Dasi, L. P., Kim, H. S., Choi, J., Leo, H. W., and Yoganathan, A. P. (2008). Structural simulations of prosthetic tri-leaflet aortic heart valves. J. Biomech. 41, 1510-1519. doi: 10.1016/j.jbiomech.2008.02.026

Halevi, R., Hamdan, A., Marom, G., Lavon, K., Ben-Zekry, S., Raanani, E., et al. (2016). Fluid-structure interaction modeling of calcific aortic valve disease using patient-specific three-dimensional calcification scans. Med. Biol. Eng. Comput. 54, 1683-1694. doi: 10.1007/s11517-016-1458-0

Hammer, P. E., Berra, I., and Nido, P. D. (2015). Surgical repair of congenital aortic regurgitation by aortic root reduction: a finite element study. J. Biomech. 48, 3883-3889. doi: 10.1016/j.jbiomech.2015.09.030

Hammer, P. E., Roberts, E. G., Emani, S. M., and Nido, P. D. (2016). Surgical reconstruction of semilunar valves in the growing child: should we mimic the venous valve? A simulation study. J. Thorac. Cardiov. Sur. 153, 389-396. doi: 10.1016/j.jtcvs.2016.08.019

Hou, Q., Liu, G., Liu, N., Pan, Y., and Qiao, A. (2019). Effect of sinus diameter on the opening and closing performance of aortic valve under the expansion of aortic root. J. Biomed. Eng. 36, 737-744. doi: 10.7507/1001-5515.201811018

Howard, I. C., Patterson, E. A., and Yoxall, A. (2003). On the opening mechanism of the aortic valve: some observations from simulations. J. Med. Eng. Technol. 27, 259-266. doi: 10.1080/0309190031000096621
Hsu, M. C., Kamensky, D., and Bazilevs, Y., (2014). Fluid-structure interaction analysis of bioprosthetic heart valves: significance of arterial wall deformation. Comput. Mech. 54, 1055-1071. doi: 10.1007/s00466-014-1059-4

Jermihov, P. N., Jia, L., Sacks, M. S., Gorman, R. C., Gorman, J. H., and Chandran, K. B. (2011). Effect of geometry on the leaflet stresses in simulated models of congenital bicuspid aortic valves. Cardiovasc. Eng. Technol. 2, 48-56. doi: 10.1007/s13239-011-0035-9

Jia, R., Ma, Y., Gu, Z., Pan, Y., Liu, S., Qiao, A., et al. (2017). Experimental study of the effects of sinotubular junction taper on artificial bioprosthesis valve in pulsatile flow condition. J. Biomed. Eng. 34, 365-370. doi: 10.7507/1001-5515.201605021

Katayama, S., Umetani, N., Sugiura, S., and Hisa-Da, T. (2008). The sinus of valsalva relieves abnormal stress on aortic valve leaflets by facilitating smooth closure. J. Thorac. Cardiov. Surg. 136, 1528-1535. doi: 10.1016/j.jtcvs.2008.05.054

Kim, H., Chandran, K. B., and Sacks, M. S., and Lu, J. (2007). An experimentally derived stress resultant shell model for heart valve dynamic simulations. Ann. Biomed. Eng. 35, 30-44. doi: 10.1007/s10439-006-9203-8

Kim, H., Lu,J., Sacks,M. S., and Chandran, K. B. (2008). Dynamic simulation of bioprosthetic heart valves using a stress resultant shell model. Ann. Biomed. Eng. 36, 262-275. doi: 10.1007/s10439-007-9409-4

Labrosse, M. R., Boodhwani, M., Sohmer, B., and Beller, C. J. (2011). Modeling leaflet correction techniques in aortic valve repair: a finite element study. J. Biomech. 44, 2292-2298. doi: 10.1016/j.jbiomech.2011.05.032

Labrosse, M. R., Lobo, K., and Beller, C. J. (2010). Structural analysis of the natural aortic valve in dynamics: from unpressurized to physiologically loaded. J. Biomech. 43, 1916-1922. doi: 10.1016/j.jbiomech.2010.03.020

Lansac, E., Bouchot, O., Arnaud, Crozat, E., Hacini, R., Doguet, F., Demaria, R., et al. (2015). Standardized approach to valve repair using an expansible aortic ring versus mechanical Bentall: early outcomes of the CA VIAAR multicentric prospective cohort study. J. Thorac. Cardiov. Surg. 149, S37-45. doi: 10.1016/j.jtcvs.2014.07.105

Laurent, D. K., Stefano, M., Munir, B., Parla, A., Jean, R., Alain, P., et al. (2014). The role of annular dimension and annuloplasty in tricuspid aortic valve repair. Eur. J. Cardio. Thorac. 49, 256-263. doi: 10.1093/ejcts/ezv050

Li, H., Pan, Y., Qiao, A., Liu, Y., Dong, N. (2019). Influence of valve height on the opening/closure performance of transplanted aortic valve. J. Biomed. Eng. 36, 199-205. doi: 10.7507/1001-5515.201709061

Marom, G., Haj-Ali, R., Raanani, E., Schafers, H. J., and Rosenfeld, M. (2012). A fluid-structure interaction model of the aortic valve with coaptation and compliant aortic root. Med. Biol. Eng. Comput. 50, 173-182. doi: 10.1007/s11517-011-0849-5

Marom, G., Haj-Ali, R., Rosenfeld, M., Schäfers, H. J., and Raanani, E. (2013). Aortic root numeric model: annulus diameter prediction of effective height and coaptation in post-aortic valve repair. J. Thorac. Cardiov. Sur. 145, 406-411. doi: 10.1016/j.jtcvs.2012.01.080

Marom, G., Hammer, P. E., Roberts, E. G., Emani, S. M., and Nido, P. J. D. (2016). Surgical reconstruction of semilunar valves in the growing child: should we mimic the venous valve? A simulation study. J. Thorac. Cardiov. Sur. 153, 389-396.

Natalie, G., Veronica, J., Holzmann, M. J., Anders, F. C., and Ulrik, S. (2016). Aortic valve replacement with mechanical vs. biological prostheses in patients aged 50-69 years. Eur. Heart. J. 34, 2658-2667. doi: 10.1093/eurheartj/ehv580

Navarra, E., Khoury, G. E., Glineur, D., Boodhwani, M., Dyck, M. V., Vanoverschelde, J. L., et al. (2013). Effect of annulus dimension and annuloplasty on bicuspid aortic valve repair. Eur. J. Cardio. Thorac. 44, 316-323. doi: 10.1093/ejcts/ezt045

Oomen, P., Holland, M. A., Bouten, C., Kuhl, E., and Loerakker, S. (2018). Growth and remodeling play opposing roles during postnatal human heart valve development. Sci. Rep-Uk. 8, 2292-2298. doi: 10.1038/s41598-018-19777-1

Pan, Y., Qiao, A., and Dong, N. (2014). Effect of the position of the coronary sinus orifice on aortic leaflet coaptation. J. Mech. Med. Biol. 14, 1-8. doi: $10.1142 / S 0219519414400090$

Pan, Y., Qiao, A., and Dong, N. (2015). Fluid-structure interaction simulation of aortic valve closure with various sinotubular junction and sinus diameters. Ann. Biomed. Eng. 43, 1363-1369. doi: 10.1007/s10439-014-1120-7

Qiao, A., Pan, Y., and Dong, N. (2014). Effects of diameters of sinotubular junction and maximum sinus on aortic valve closure. J. B. Univ. Technol. 40, 776-780. 
Ravil, S., Alexander, B. P., Sergey, Z., Igor, D., Alexey, P., Alexander, A., et al. (2019). Factors impacting long-term pulmonary autograft durability after the ross procedure. J. Thorac. Cardiov. Surg. 157, 134-141. doi: 10.1016/j.jtcvs.2018.05.046

Rim, Y., Mcpherson, D. D., and Kim, H. (2014). Mitral valve function following ischemic cardiomyopathy: a biomechanical perspective. Biomed. Mater. Eng.24, 7-13. doi: 10.3233/BME-130777

Schäfers, H. J., Schmied, W.,Marom, G., and Aicher, D. (2013). Cusp height in aortic valves. J. Thorac. Cardiov. Surg. 146, 269-274. doi: $10.1016 /$ j.jtcvs.2012.06.053

Wazaren, H., Bouhdadi, H., Boussaadani, B. E., Rhissassi, J., and Laaroussi, M. (2021). Use of neocords in the treatment of mitral valve prolapse: about 6 cases. Int. J. Surg. 80:105693. doi: 10.1016/j.ijscr.2021.105693

Weinberg, E. J., and Mofrad, M. R. (2007). Transient, three dimensional, multiscale simulations of the human aortic valve. Cardiovasc. Eng. 7, 140-155. doi: 10.1007/s10558-007-9038-4

Weltert, L., Tullio, M. D. D., Afferrante, L., Salica, A., Scaffa, R., Maselli, D., et al. (2013). Annular dilatation and loss of sino-tubular junction in aneurysmatic aorta: implications on leaflet quality at the time of surgery. A finite element study. Interact. Cardiov. Ther. 17, 8-12. doi: 10.1093/icvts/ivt116

Zhang, R., Xie, J., Zhou, J., Xu, L., and Yang, Y. (2021). Supravalvular aortic stenosis and the risk of premature death among patients with homozygous familial hypercholesterolemia. Am. J. Cardiol. 145, 58-63. doi: 10.1016/j.amjcard.2020.12.080

Conflict of Interest: The authors declare that the research was conducted in the absence of any commercial or financial relationships that could be construed as a potential conflict of interest.

Publisher's Note: All claims expressed in this article are solely those of the authors and do not necessarily represent those of their affiliated organizations, or those of the publisher, the editors and the reviewers. Any product that may be evaluated in this article, or claim that may be made by its manufacturer, is not guaranteed or endorsed by the publisher.

Copyright (c) 2021 Hou, Liu, Liu, Zhang, Qu, Zhang, Li, Pan and Qiao. This is an open-access article distributed under the terms of the Creative Commons Attribution License (CC BY). The use, distribution or reproduction in other forums is permitted, provided the original author(s) and the copyright owner(s) are credited and that the original publication in this journal is cited, in accordance with accepted academic practice. No use, distribution or reproduction is permitted which does not comply with these terms. 